\title{
Perceptions of Personal Health Records in Rural Primary Health Clinics in Canada- Patient Perspectives
}

\author{
Leanne M CURRIE ${ }^{\mathrm{a}, 1}$, Kathy RUSH ${ }^{\mathrm{b}}$, Lindsay BURTON ${ }^{\mathrm{b}}$, \\ Mona MATTEI ${ }^{\mathrm{c}}$ and Matthias GÖRGES ${ }^{\mathrm{d}}$ \\ ${ }^{a}$ School of Nursing, University of British Columbia-Vancouver, BC, Canada \\ ${ }^{\mathrm{b}}$ School of Nursing, University of British Columbia-Okanagan, Kelowna, BC, Canada \\ ${ }^{\mathrm{c}}$ Kootenay Boundary Division of Family Practice, Grand Forks, BC, Canada \\ ${ }^{\mathrm{d}}$ Department of Anesthesiology Pharmacology \& Therapeutics, University of British \\ Columbia, and Research Institute, BC Children's Hospital, Vancouver, BC, Canada
}

\begin{abstract}
Personal health records are increasingly being deployed in healthcare settings. In this study we explored patients' perceptions of personal health records in a rural community in Canada where a primary health network is being deployed. A focus group was held and data were thematically analysed. All patients used technology on a regular basis. Themes included communication and information sharing, issues with access to prior health records, data content and data control and features and functions for continuity of care. Participants expressed desire to be owners of their own record, but described instances where they might be too ill to do so. Participants were hopeful that the functions of a personal health record might help to overcome frustrations with current fragmented information and open to using technologies as part of their care process. Personal health records are promising technologies to overcome fragmented care in rural communities.
\end{abstract}

Keywords. Nursing Informatics, rural, personal health record, patient oriented research, patient reported outcomes measures

\section{Introduction}

Personal health records and patient portals are increasingly being deployed across healthcare settings [1]. The overall goals of these systems are to support the vision of a lifelong record of a person's health and illness data, provide a record of interactions with healthcare providers and the healthcare system, and often provide methods for secure communication with healthcare providers [1]. In a recent systematic review about the use of PHRs, the authors found that sex and health status did not affect use of PHRs but that higher income level and greater awareness increased use of PHRs [1]. Less is known about how these tools work in rural communities where there is evidence that geographical disparities affect how people engage in chronic illness care [2].

Several international initiatives have been focusing on patient reported outcome measures (PROMS) as a method to ensure that the patient perspective is captured in

${ }^{1}$ Corresponding Author, Leanne M Currie, University of British Columbia, School of Nursing, Vancouver, BC, V5T 2B5, Canada; E-mail: leanne.currie@ubc.ca. 
healthcare encounters, particularly for chronic illness management [3]. While these initiatives are growing, it is not clear who should be managing the data related to a patients' chronic illnesses [4] and less understanding about patients' values related to sharing their data. The purpose of this study was to explore rural patients' perceptions of PHRs, their preferred features and functions of PHRs and to understand their perceptions about sharing personally generated data with healthcare providers.

\section{Mthods}

In this paper we report on Phase 1 of a mixed-methods study that is exploring the use of PHRs in a rural context where a primary care network infrastructure is being deployed. Prior to data collection, the study was approved through the local university research ethics board and the local healthcare institution. Patients were recruited through a patient advisory committee which was convened by the local health authority. The group meets on a monthly basis. Some members have been with the group for several years, while others have only joined recently.

\subsection{Setting}

The study was carried out in a rural region in British Columbia, Canada. This region is mountainous and relatively sparsely populated with a large proportion of inhabitants over the age of 65 years, and many with fixed incomes [5]. In addition, the region has several First Nations communities who receive healthcare from the provincial healthcare services. Canada has universal healthcare coverage for all citizens, and in this region, while the distances may not be far, mountain passes are frequently snowbound making access to clinicians tenuous at times [6]. In addition, some regions have poor cellular network reception and less options for internet access [6].

\subsection{Data Collection and Analysis}

Prior to starting the focus group, all participants completed a demographics survey and provided informed consent. The meeting room was set in a $\mathrm{u}$-shaped format and individuals were assigned numbers and asked to state their number prior to speaking. Participants were also asked to speak one at a time. One researcher (LC) lead the focus group and other members of the research team took notes or were responsible for the demonstration of the a PHR software that will be used in the region. The following questions guided the session: 1. Tell us about your understanding of Personal Health Records? 2. What is your perception of the value of using PHRs in your daily life? 3. What types of communication methods do you think you would prefer to use with your healthcare provider team? 4. Tell us about your interpretation of 'patient-generated data'. How are your data different from data gathered by your healthcare team, if at all? 5 . What does it mean to you to share your personally generated data with your healthcare providers? After these questions were discussed, a demonstration of an example PHR was provided for participants. To conclude the focus group the final question was asked: 6. What is most important to you about sharing your personal data with the healthcare team?. The focus group lasted 90 minutes. At the end of the session participants were provided with an honorarium of $\$ 25 \mathrm{CAD}$. The meeting was audio-recorded and 
transcribed verbatim. Field notes were taken by team members during the session. Thematic analysis was carried out on the transcripts and field notes.

\section{Results}

\subsection{Demographics}

Nine patients participated in the focus group ( 8 female, 1 male). Ages ranged from 28 to 82 with a mean age of $59.4(\mathrm{SD}=17.7)$. Three of the members identified as Indigenous while all other participants identified as Caucasian. Most had a college education $(n=6$, $67 \%$ ), 2 had some college, and one had high school education. There were a wide range of household incomes with 3 participants having incomes of $<\$ 25 \mathrm{~K}, 2$ with incomes of $\$ 25 \mathrm{~K}-\$ 50 \mathrm{~K}, 2$ with incomes of $\$ 51 \mathrm{~K}-\$ 75 \mathrm{~K}$, and 2 with incomes of $>\$ 75 \mathrm{~K}$. All lower than the median income in $\mathrm{BC}$ of $\$ 86.5 \mathrm{~K}$. Distance from their primary care provider ranged from $1 \mathrm{Km}$ to $25 \mathrm{Km}$ (M 5.7, SD 7.5).

One participant reported no chronic health problems, 2 reported 1 chronic health problem, 2 reported 2 chronic health problems, and 3 reported 3 chronic health problems. Three participants took no medications, 3 took 1-2 medications, and 3 took 3-4 medications. Participants reported their overall health to be excellent $(n=3)$, fair $(n=3)$ or good $(n=3)$, and overall mental health to be excellent $(n=5)$, fair $(n=3)$ or $\operatorname{good}(n=1)$. Lastly, all participants used technology: five of the participants used technology 'always' and four used technology 'regularly.'

\subsection{Thematic Analysis}

In response to the first question about awareness of personal health records, there was overall lack of knowledge about the availability of such technologies, and participants were somewhat surprised that these might be offered through a primary care provider. Several themes arose during the focus group discussion including: communication and information sharing, issues with prior health records, data content and data control, and functions to support continuity of care and patient education.

\subsection{Communication and Information Sharing}

Participants noted that they were surprised that all members of the healthcare team didn't already have access to all of their information. This resulted in frustrations with disconnection of medical information across healthcare settings and the need to frequently repeat information to each provider. For example, when asked what was most important about sharing data with healthcare providers one participant commented:

I think just not repeating the same thing over and over again to different people and what you have is, like, triggering for a lot of things. I mean, you could potentially go forward way faster instead of having to relapse all the time going back into the story and someone... and getting sent to somebody else, so it would just be one voice.

After the functions of the PHR were presented to the group, there was overall optimism that access to the PHR may resolve some of these issues. 


\subsection{Issues with Prior Health Records}

Several participants reported that prior healthcare information was missing or not accessible, not acted on or disagreed with by a new care provider, or not understandable to the patients. Patients reported difficulty accessing information from previous healthcare providers, and wondered if a PHR would solve this problem. One participant reported that the provider 'refused' to send their records to the new provider which meant that the patient needed to repeat themselves to provide their history. Another participant noted that even though the provider had access to the record from a different Canadian province "[the new provider] would not look at [the previous files]." Others in the group noted that even when they received the records they (the patients) were unable to read the providers' notes: "I couldn't read a word of it, so to me that means I think there needs to be some type of middle terminology for layman's terms." One participant mentioned that her foster childrens' family histories were not contained in current records, thus they did not know if the children were at risk for certain disorders.

Another issue that arose was related to the regional context. Participants reported that there are transient people, such as those who come for seasonal work, people who are arriving from other provinces in Canada, women in transition (from unsafe living environment to a safe environment), and people with post-traumatic stress. Participants worried that because of the transient nature of these peoples' lives, the current systems were "extra challenging for transient people", particularly because the transient nature of these individuals' lives resulted in having multiple disparate care providers.

\subsection{Data Content and Data Control}

Participants stated that they value sharing their data with clinicians, but were concerned that if the data were not 'all inclusive', that missing information might be a barrier to receiving adequate healthcare services. Indeed, many participants stated that the record should be owned by the patient. One participant stated:

....if you [the patient] give permission, then you should be able to sit and be

the gatekeeper and just say, you know, "Here's the access to my file, you know,

go for it." And so, that would transfer the information without having to go

through some kind of authority. If it's your file, you should be able to do that.

Multiple participants commented that they were worried that they might be ill and therefore not well enough to manage their own records and would want someone else to be a proxy. For example: patients who are not in a mental state to manage their own record, children who are in foster care, and elderly parents who are being cared for by their middle-aged children.

\subsection{Features and Functions for Continuity of Care and Patient Education}

Participants were overall pleased with the features and functions demonstrated (secure messaging, video conference appointments, pre-visit surveys). There was general consensus that use of the PHR would require patient education and concern about who would bear the costs of using the PHR. For example, would the patient need a subscription? Would the healthcare system pay for Internet access? Regarding secure communication with providers, they indicated they would like to choose whether they received information via an email or a text. While they liked the idea of completing pre- 
visit surveys, they hoped that the provider would review the surveys before their appointment so that the patient did not have to repeat themselves.

\section{Discussion}

In this study we explored community members' perspectives about use of a PHR. All members of the group use technology regularly or daily which is higher than reported in Canadian rural statistics [7]. However, the group had a lower income than the median for BC. There was general optimism about adopting PHRs in a rural context and willingness to share personal health data with the care team and other relevant individuals. The main worries were related to inappropriate sharing of the information and costs associated with using such systems.

Two of the main themes were related to disconnected information in current computer systems resulting from people moving across different healthcare providers and providers appearing to not trust information gleaned from other clinicians. Indeed, participants were surprised that their information was not shared across healthcare teams. Given that the patient may have changed between the time points, clinicians are likely being prudent, but this can manifest as a burden on the patient. While patients valued sharing data with and between providers, participants wanted to ensure that only the appropriate people had access to those data. There were two main limitations to the study. Firstly, the focus group was an existing known group of people which, although may have facilitated dialogue, also may have inhibited some members due to unknown existing relationships. Secondly, overall, this participant group were highly educated, had moderately high incomes, and had a small number of chronic illnesses.

\section{Conclusions}

PHRs are promising solutions to facilitate illness management and support care coordination in rural communities. Patient perspectives are valuable to guide implementation of PHR systems.

\section{References}

[1] Abd-alrazaq AA, Bewick BM, Farragher T, Gardner P. Factors that affect the use of electronic personal health records among patients: A systematic review. International Journal Of Medical Informatics 126 (2019), 164-175.

[2] Smith ML, Towne Jr SD, Bergeron CD et al. Geographic disparities associated with travel to medical care and attendance in programs to prevent/manage chronic illness among middle-aged and older adults in Texas. Rural And Remote Health 19 (2019), 5147-5147.

[3] Hjollund NH. Fifteen years' use of patient-reported outcome measures at the group and patient levels: Trend analysis. Journal Of Medical Internet Research 21 (2019), e15856-e15856.

[4] Ancker JS, Witteman HO, Hafeez B, Provencher T, Van de Graaf M, Wei E. The invisible work of personal health information management among people with multiple chronic conditions: qualitative interview study among patients and providers. Journal Of Medical Internet Research 17 (2015), e137e137.

[5] Statistics Canada. Census Profile, 2016 Census - Kootenay Boundary, Regional district [Census division], British Columbia and British Columbia [Province]. In, 2016.

[6] Government of Canada. National broadband internet service availability map. In, 2019.

[7] Government of Canada. High-speed access for all: Canada's connectivity strategy. In, 2017. 